\title{
A Flexible Platform for Biofeedback-driven Control and Personalization of Electrical Nerve Stimulation Therapy
}

\author{
Matthew P. Ward, Kurt Y. Qing, Kevin J. Otto, Member, IEEE, Robert M. Worth, Simon W.M. John, \\ and Pedro Irazoqui, Member, IEEE
}

\begin{abstract}
Electrical vagus nerve stimulation is a treatment alternative for many epileptic and depressed patients whose symptoms are not well managed with pharmaceutical therapy. However, the fixed stimulus, open loop dosing mechanism limits its efficacy and precludes major advances in the quality of therapy. A real-time, responsive form of vagus nerve stimulation is needed to control nerve activation according to therapeutic need. This personalized approach to therapy will improve efficacy and reduce the number and severity of side effects. We present autonomous neural control, a responsive, biofeedbackdriven approach that uses the degree of measured nerve activation to control stimulus delivery. We demonstrate autonomous neural control in rats, showing that it rapidly learns how to most efficiently activate any desired proportion of vagal $A$, B, and/or $C$ fibers over time. This system will maximize efficacy by minimizing patient response variability and by minimizing therapeutic failures resulting from longitudinal decreases in nerve activation with increasing durations of treatment. The value of autonomous neural control equally applies to other applications of electrical nerve stimulation.
\end{abstract}

Index Terms-Biofeedback, electrical stimulation, neural control, neuroprosthesis

\section{INTRODUCTION}

$\mathrm{V}$ AGUS nerve stimulation (VNS) is a treatment alternative for many epileptic and depressed patients whose symptoms are not well managed with pharmaceutical therapy. Approximately 2-weeks after device implantation, a physician programs the pacemaker-like device to deliver intermittent pulses of current to the left cervical vagus nerve. The highest efficacy is typically observed after 1 year, but

Manuscript received November 12, 2013; revised May 30, 2014; accepted August 10, 2014. Date of publication XXX XX, 2014; date of current version August 20, 2014. This work was supported in part by Cyberonics, Inc.

M. P. Ward, K. Y. Qing, and P. P. Irazoqui are with the Department of Biomedical Engineering at Purdue University, West Lafayette, IN 47907 USA. P. P. Irazoqui is also with the Department of Electrical and Computer Engineering at Purdue University, West Lafayette, IN 47907 USA (e-mail: mpward@purdue.edu; kqing@purdue.edu; pip@purdue.edu).

K. J. Otto is with the Department of Biomedical Engineering at the University of Florida, Gainesville, FL 32611 USA (e-mail: kotto@purdue.edu).

R. M. Worth is with the Department of Neurological Surgery at the Indiana University School of Medicine, Indianapolis, IN 46202 USA. He is also with the Department of Mathematical Sciences at the Indiana University-Purdue University of Indianapolis, Indianapolis, IN 46202 USA (e-mail: rworth@iupui.edu).

S. W. M. John is with the Howard Hughes Medical Institute at the Jackson Laboratory, Bar Harbor, ME 04609 USA (e-mail: simon.john@jax.org). only after several minimally informed stimulus parameter adjustments $[1,2]$. The efficacy of these treatments is far from optimal.

Over the course of weeks to months, a physician systematically tunes the stimulus until the patient and physician feel that the therapy is working with no adverse or intolerable side effects. If a bothersome side effect is encountered, the intensity of stimulation is decreased until the side effect disappears. These parameters are maintained until the next appointment $[1,2]$. Major limitations beyond the subjective nature of this approach include 1) the risk of adaption or desensitization to the stimulus, which may make the therapy less effective over time (e.g., stimulus induced depression of neuronal excitability, or SIDNE [3]), 2) the lack of feedback regarding the type and number of neurons that are activated when the therapy is effective, and 3) the risk of patient discomfort [1].

All electrical nerve stimulation (ENS) therapies use some form of a stimulus parameter-based dosing system. This is problematic, as stimulus parameters are poor predictors of therapeutic efficacy; each patient and nerve responds uniquely to the same strength of stimulation, and the relationship between stimulation and the degree of nerve activation changes over time. These factors limit treatment benefit and contribute to poorer efficacy on a shorter timescale [2]. They also help to explain why the therapeutic mechanisms are not well understood despite decades of investigation [1]. An objective, informed dosing system is required to improve the efficacy of ENS therapies and to further reduce the number and severity of side effects.

We present autonomous neural control (ANC), a nerve activation control system designed to eliminate patient response variability and the detrimental effects of the foreignbody response at the device-tissue interface. In rats, ANC rapidly learns how to most efficiently activate any proportion of vagal $\mathrm{A}, \mathrm{B}$, and/or $\mathrm{C}$ fibers over time. It provides a new dosing mechanism based on neural activation. In real time, ANC systematically decodes evoked compound nerve action potential (CNAP) responses to construct a patient-specific nerve activation profile (NAP), which describes how each neuron population in the nerve will respond to any strength of stimulation. Over the course of ENS therapy, ANC continuously refines the NAP to improve its prediction accuracy and adapt to circadian, drug-induced, or immunemediated changes at the device-tissue interface (Fig. 1) [4]. 


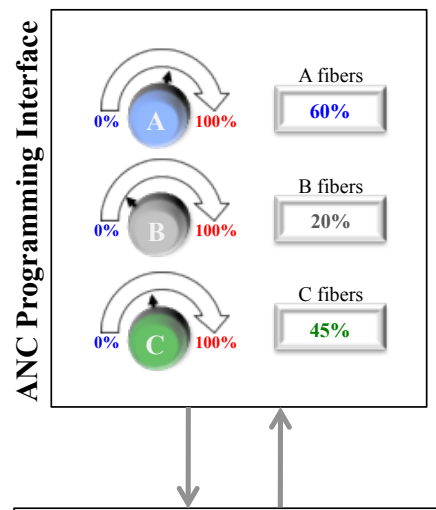

\begin{tabular}{|l|l}
\hline Addiction \\
Alzheimer's Disease \\
Anxiety Disorders \\
Generalized Anxiety Disorder \\
Obsessive-compulsive Disorder \\
Panic Disorder \\
Post-traumatic Stress Disorder \\
Social/Specific Phobia \\
Autoimmune Inflammatory Disorders \\
Crohn's Disease \\
Rheumatoid Arthritis \\
Systemic Lupus Erythematosus \\
Ulcerative Colitis
\end{tabular}

Chronic Pain
Failed Back Surgery Syndrome
Neuropathic Pain
Radiculopathy
Depression
Diabetes
Epilepsy
Heart Failure/Cardiac Arrhythmia
Atrial Fibrillation
Congestive Heart Failure
Migraine
Motor/Movement disorders
Nerve Regeneration

Obesity

Obstructive Sleep Apnea

Reactive Airway Disease Asthma

Bronchoconstriction

COPD

Exercise-induced

Bronchospasm

Sensory Disorders

Sepsis

Stroke Rehabilitation

Tinnitus

Urinary Incontinence

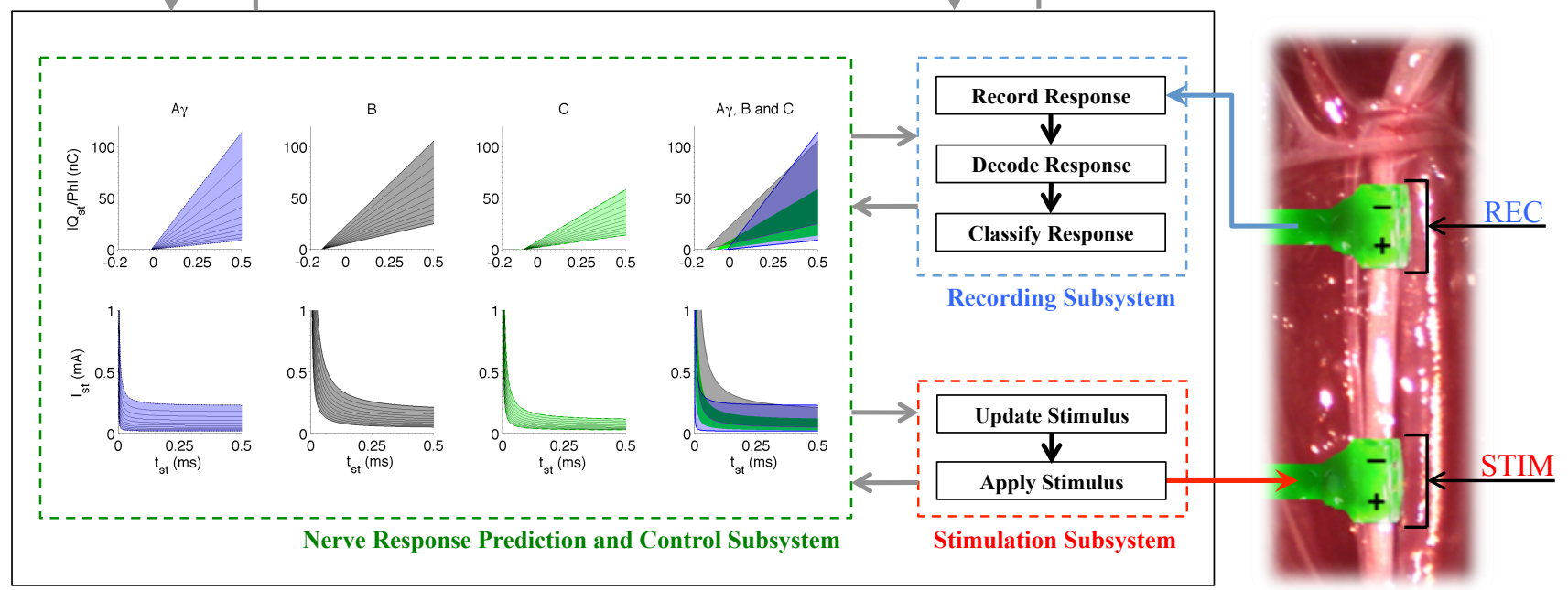

The Autonomous Neural Control (ANC) System

Fig. 1. Summary and applications of ANC. ANC enables rapid, consistent control over the biological conduits of therapy. Using features from the CNAP, ANC rapidly constructs a NAP to predict how one or more neuron populations in a nerve will respond to any rectangular, constant-current stimulus pulse. The NAP enables dial based-control over the biology, simplifying device optimization and standardizing therapeutic, investigational and data reporting methods.

ANC refines the electrical stimulus, within safe limits [3, 57], to selectively control nerve activation on a patient-topatient, nerve-to-nerve and neuron-to-neuron basis. By providing consistent nerve activation, ANC allows reproducible experiments to systematically delineate the therapeutic mechanisms of VNS or other form of ENS therapy. Furthermore, biological markers of treatment response may be measured and classified with respect to the NAP, simplifying the development of fully personalized, closed-loop control systems for treating diverse neurological diseases.

For physicians, ANC will 1) establish an objective, standardized dosing system based on the level of nerve/neuron activation or inhibition, expressed as a \% of maximal nerve/neuron activation, 2) eliminate the complicated, timeconsuming stimulus parameter tuning process, 3) provide a simple mechanism to adjust the relative ratios of $\mathrm{A}, \mathrm{B}$ and $\mathrm{C}$ fiber activation, and 4) ensure that therapeutic nerve/neuron activation is maintained over time. For patients, ANC will 1) improve efficacy and enhance the overall quality of ENS therapy, 2) reduce the number of doctor visits, and 3) help extend device lifetime by reducing energy waste from excessive stimulation.

\section{MethodS}

\section{A. Surgical Methods}

All surgical and animal handling procedures are approved by the Institutional Animal Care and Use Committee (IACUC) and adhere to guidelines set forth in the Guide for the Care and Use of Laboratory Animals [8]. All rats are housed in a 12-hr light/dark cycle at constant humidity and temperature. The surgical suite and instruments are sterilized prior to each procedure. Isoflurane gas anesthesia is used for the duration of surgery; it is set to the lowest level that will maintain a stable anesthetic plane $\left(0.5-3 \%\right.$ isoflurane in $\left.2 \mathrm{~L} / \mathrm{min}_{2}\right)$. Following induction, the rat is placed in a supine position, the surgical site is shaved and cleaned with alternating scrubs of betadine and $70 \%$ ethanol, and an analgesic is provided (butorphanol tartrate; $0.5-2 \mathrm{mg} / \mathrm{kg}, \mathrm{SC}$ ). A small support is placed below the neck for stability. Throughout the procedure, subcutaneous fluids are provided as needed to prevent dehydration.

Once the surgical site is clean, a 1.5 to $2 \mathrm{~cm}$ long midline incision is made from the jaw line to manubrium. A blunt dissection technique is used for the remaining steps of the procedure. With a pair of curved, blunt-tipped scissors, we tunnel through the subcutaneous tissues until the sternohyoid, 
omohyoid, and sternocleidomastoid muscles are visible. The connective tissue between the left sternocleidomastoid and the sternohyoid/omohyoid is carefully separated until the carotid sheath is visible. Using a small pair of surgical retractors to hold the muscles apart, the vagus nerve and carotid artery are dissected from the carotid sheath. After isolating a 1.0 to 1.5 $\mathrm{cm}$ segment of the cervical vagus nerve, the epineurium is carefully pierced with iris scissors and retracted with fine forceps.

Two custom-made silicone cuff electrodes are wrapped around the nerve; care is taken to ensure circumferential or near-circumferential contact with the nerve (Electrode spacing $=1 \mathrm{~mm}$; Electrode surface area $\cong 3 \mathrm{~mm}^{2}$ ). The stimulation leads are connected to the output of an A-M Systems Model 2200 Analog Stimulus Isolator. The recording leads are connected to the inputs of a Grass Model P511 High Performance AC Preamplifier. The stimulation leads are connected such that the cathode (-) is closest to the noninverting recording electrodes. A data acquisition board (National Instruments USB-6353 X Series) is used to interface with a computer running ANC in Matlab R2010a.

\section{B. Stimulus Artifact Suppression}

A limited CNAP conduction distance is available along the left cervical vagus nerve of rodents (e.g., $\sim 5-15 \mathrm{~mm}$ of exposed nerve in a 280-300 g rat). As a result, CNAP response peaks often coincide with the stimulus artifact, necessitating the use of an artifact suppression method [9]. To the best of our knowledge, we are the first to demonstrate effective and reliable stimulus artifact suppression using cathode-first, alternating monophasic stimulation in the peripheral nervous system at conduction distances less than $1 \mathrm{~cm}$.

Fig. 2 summarizes the method of CNAP extraction and averaging using the measured response to a 1-s train of cathode-first, alternating monophasic stimulation $\left(I_{s t}=\right.$ stimulus pulse amplitude $=0.058 \mathrm{~mA} ; t_{s t}=$ stimulus pulse width $=0.4 \mathrm{~ms} ; P R F=$ pulse repetition frequency $=20 \mathrm{~Hz}$; $t_{\text {train }}=$ stimulus train duration $=1 \mathrm{~s} ; F s=$ sampling frequency $=$ $50 \mathrm{kHz}$; pass-band $=0.001$ to $10 \mathrm{kHz}$ ). The raw cathodal and anodal stimulus artifacts are shown in black and grey, respectively. To remove any DC offset, the average of the raw response waveform is subtracted from the recording. Then, ANC segments the raw response waveform into $N$ periods of responses $\left(N=P R F^{*} t_{\text {train }}=20\right)$. Each period of the response waveform is then further segmented and grouped into clusters of cathodal and anodal response waveforms, respectively (Fig. 2B). The cathodal and anodal stimulus artifacts are symmetric in the recordings due to the symmetry of the anodal and cathodal phases of stimulation and the natural orientation of the recording electrodes along equipotential lines of the electric field radiating from the stimulating electrodes. The sum of each anodal and cathodal response waveform yields a cluster of artifact-free vagal nerve responses to stimulation (Fig. 2C). The mean CNAP response waveform (Fig. 2E) is the averaged cluster of CNAP responses in Fig. $2 \mathrm{C}$ and the sum of the mean cathodal and anodal response waveforms in Fig. 2D. A Shapiro-Wilk test for normality gives no evidence that the artifact-free stimulus responses are not normally

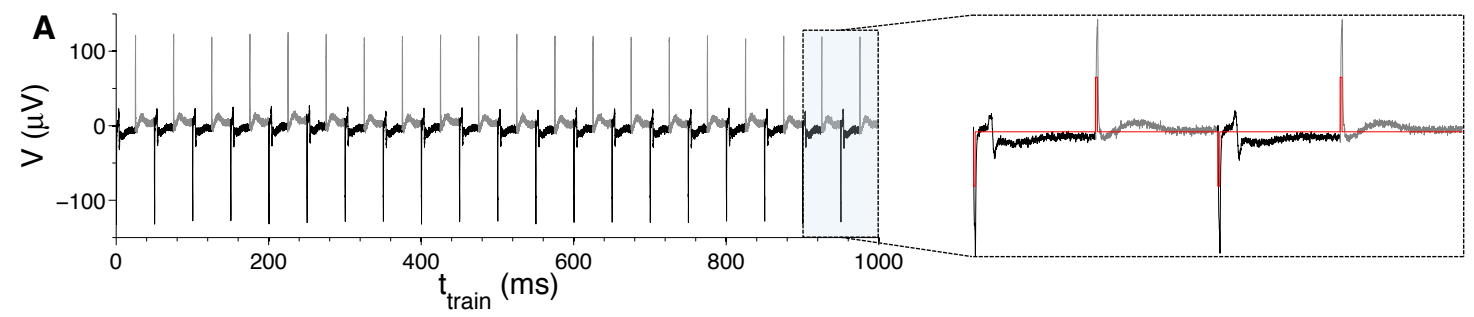

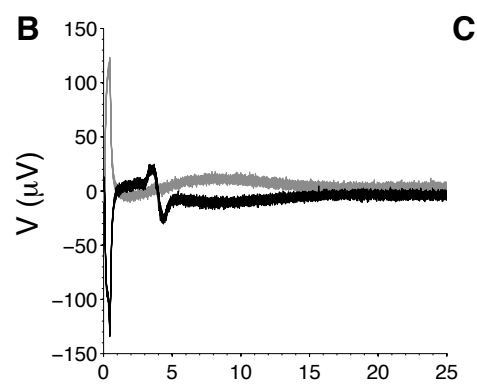
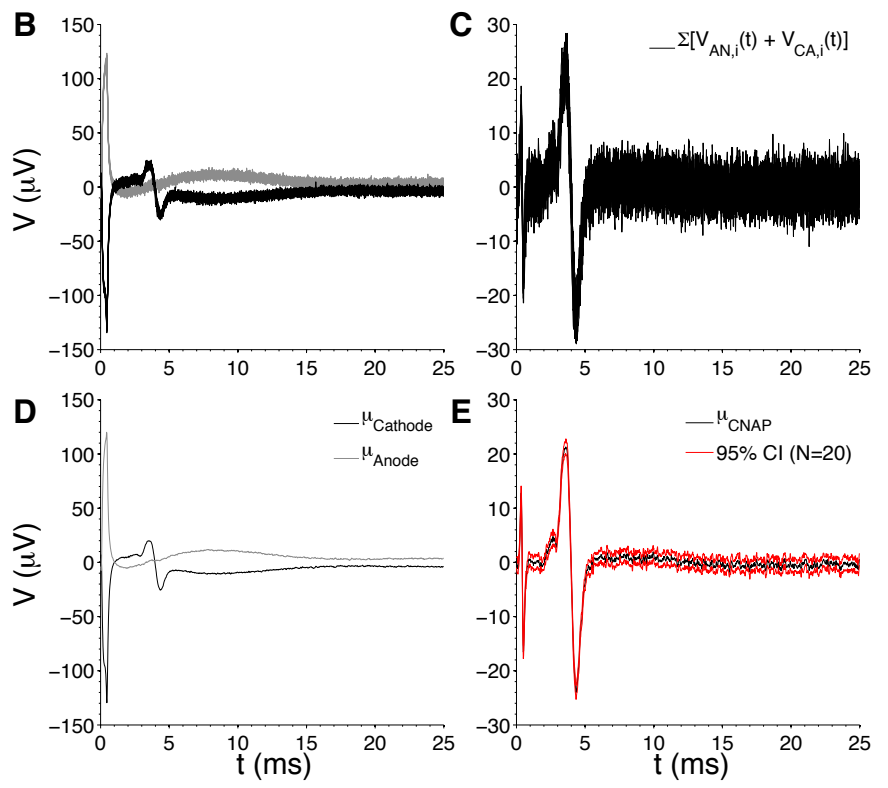

Fig. 2. Summary of the cathode-first, alternating-monophasic stimulation method used by ANC [9]. ANC suppresses stimulus artifacts via a symmetric stimulation method in which the cathodal and anodal phases of stimulation have an identical shape and opposite polarity. In most cases, nerve fibers are only activated in response to the cathodal pulse (the inset overlays the stimulus waveform in red). ANC clusters each cathodal and anodal response to a train of biphasic stimuli (A), clusters the cathodal and anodal response waveforms (B), computes the artifact-free responses to each period of stimulation by summing the cathodal and anodal responses within a period of stimulation (C), computes the mean cathodal and anodal response waveforms (D), and sums the resulting waveforms to yield the mean CNAP response (E). The 95\% confidence interval is shown in red in (E). 
distributed $(\mathrm{Prob}>\mathrm{z}=0.889$ when stimulating at $20 \mathrm{~Hz}$ for 1 s). Therefore, a response at any point in the signal is significantly different from $0 \mathrm{~V}$ (at $\alpha=0.05$ ) if the mean response and $95 \% \mathrm{CI}$ do not cross the abscissa.

\section{Nerve Response Classification}

ANC deconstructs the stimulus-evoked CNAP, recorded at a fixed distance from the stimulating cathode, to estimate the level and type of nerve fiber activation. Conduction velocity is used to identify distinct nerve fiber groups (i.e., neuron populations), referred to as A (fast, myelinated fibers), B (slow, myelinated fibers), or C (slow, unmyelinated fibers) $[10,11]$. Fig. 3 describes this Letter System using data collected from the left cervical vagus nerve of a female LongEvans rat. When recording at a fixed, known distance from the stimulating cathode, the CNAP response waveform peaks separate in time due to the differing conduction velocities of $\mathrm{A}, \mathrm{B}$ and $\mathrm{C}$ fibers. The maximal CNAP response, otherwise referred to as maximal activation, is the CNAP response magnitude at which an increase in stimulus intensity does not produce an increase in response. By individually deriving stimulus-response relationships for $\mathrm{A}, \mathrm{B}$ and $\mathrm{C}$ fibers, the effect of any stimulus pulse on nerve activity is directly measurable. Before ANC, however, this information has not been readily available or exploited in therapeutic applications.
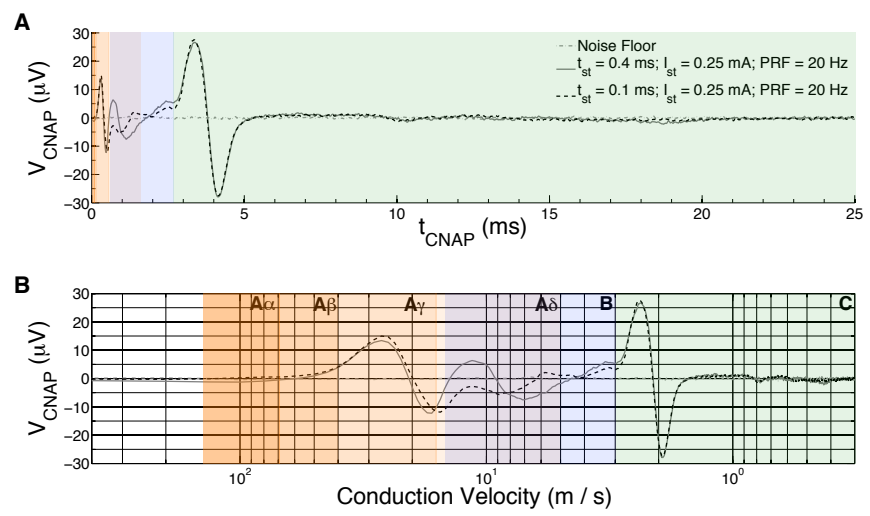

Fig. 3. CNAP classification system built into ANC. (A) Mean CNAP response from the left cervical vagus nerve of rat. Shaded regions in (A) correspond to a conduction velocity range in (B), which enables nerve fiber classification according to the Letter System [15]. (B) Mean CNAP response from (A) plotted as a function of conduction velocity, in $\mathrm{m} / \mathrm{s}$ (Conduction Distance $=$ $8.0 \pm 0.5 \mathrm{~mm})$.

\section{Stimulus-Response Measurement and Classification}

ANC measures a series of stimulus-response relationships to construct an empirical model that describes how each fiber type in any nerve of any patient will respond to any strength of electrical stimulation. This model, known as a nerve activation profile, describes the sensitivity and dynamic range of each fiber type that can be identified in a CNAP. It can be constructed in under a minute. ANC continuously updates the NAP to improve its prediction accuracy over time and adapt to the variety of factors that influence the efficacy of stimulation (e.g., circadian effects, changes in the electrode-tissue interface over time, or fiber desensitization to stimulation). By controlling nerve activation through dynamic adjustments to stimulus charge, accomplished through NAP-guided changes in stimulus pulse amplitude or width, ANC enables ENS with a constant nerve activation effect in each patient. This enables much more consistent therapy.

The sensitivity of each fiber group to ENS is evaluated using stimulus-response data collected at $t_{s t}=0.4,0.2$ and 0.1 ms. If necessary, the operator may define all stimulus and recording parameters (default parameters: stimulus type $=$ constant current; stimulus waveform $=$ cathode-first, alternating monophasic stimulation; $P R F=20 \mathrm{~Hz} ; t_{\text {train }}=1 \mathrm{~s}$; $F s=50 \mathrm{kHz}$; pass-band $=0.001$ to $10 \mathrm{kHz}$ ). Starting with $t_{s t}=$ $0.4 \mathrm{~ms}$, ANC incrementally increases the stimulus amplitude, stimulates the nerve, and records that resulting CNAP response. Between trials, the mean CNAP response is computed, the peak fiber responses are located and classified, and the data are stored in local memory. Following Trial 1, the response magnitude from the target fiber group is always compared to that from the previous trial. When stimulus intensity is increased and the target fiber response magnitude no longer increases (i.e., if a fiber group is maximally activated), ANC stores the stimulus parameters and responses from the previous trial. Next, ANC decreases the stimulus amplitude according to (1) until parameters that yield a predefined percentage of maximal activation are located (e.g., $25 \%$ maximal activation, defined as a target fiber response having a magnitude that is $25 \%$ of its maximal response magnitude). The same process is repeated using $t_{s t}=0.2$ and $0.1 \mathrm{~ms}$, respectively. An error tolerance of $5 \%$ is initially used to classify all fiber response magnitudes to account for the effects of noise.

$$
Q_{n}=Q_{n-1}\left(1+\frac{V_{\odot}-V_{C N A P, n-1}}{k \cdot V_{\odot}}\right)
$$

In (1), $Q_{n-1}$ is the stimulus charge per phase from the most recent trial (in $\mathrm{C} / \mathrm{Ph}$ ), $V_{\odot}$ is the target fiber response voltage (in V), $V_{C N A P, n-1}$ is the fiber response voltage from the most recent trial, and $k$ is a scaling factor that modulates the magnitude of the stimulus intensity adjustment (e.g., when $\mathrm{k}$ is greater than unity, it reduces the intensity of the stimulus charge adjustment; when $\mathrm{k}$ is less than unity, it amplifies the intensity of the stimulus charge adjustment). The new stimulus pulse amplitude is calculated by dividing the new stimulus charge per phase, $Q_{n}$, by the pulse duration used in the preceding trial (i.e., $I_{s t, n}=Q_{n} / t_{s t, n-1}$ ).

\section{RESULTS}

\section{A. Rapid Loss of C-fiber Activation with Constant Stimulation}

Fig. 4 shows an example of how a nerve adapts to a constant electrical stimulus in a relatively short time $\left(I_{s t}=0.2\right.$ $\left.\mathrm{mA} ; t_{s t}=0.5 \mathrm{~ms} ; P R F=20 \mathrm{~Hz} ; t_{\text {train }}=30 \mathrm{~s}\right)$. Suppl. Fig. 1 


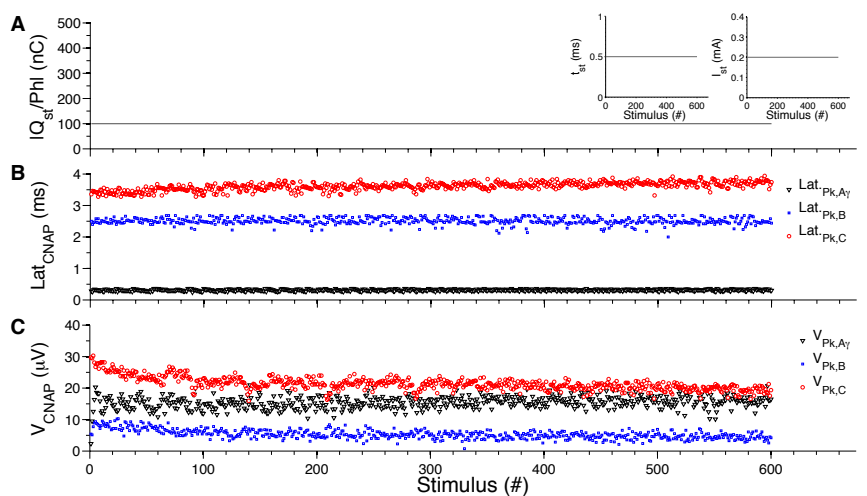

E

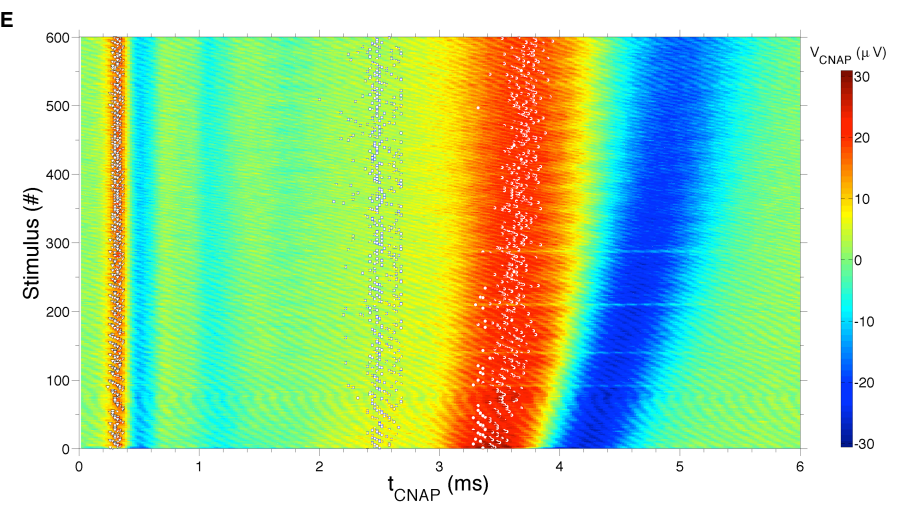

D

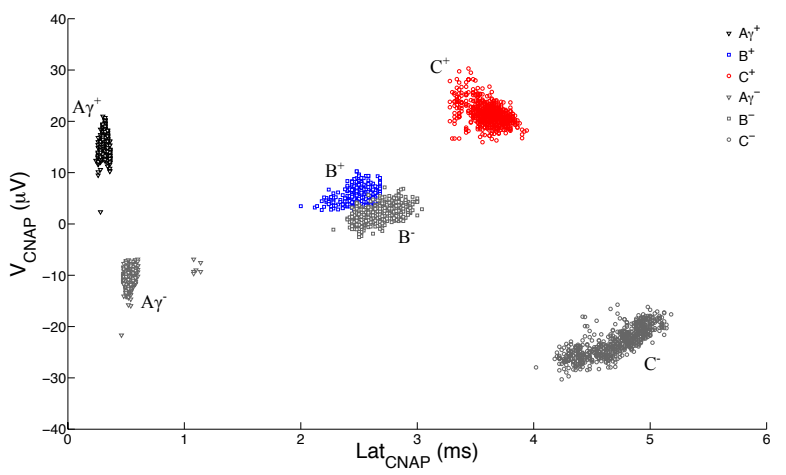

$F$

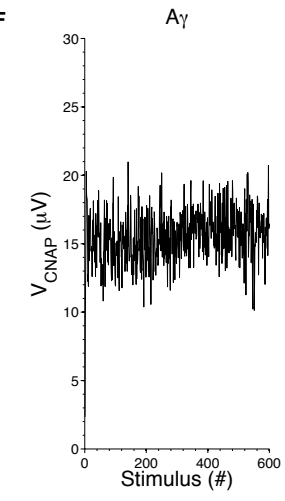

B

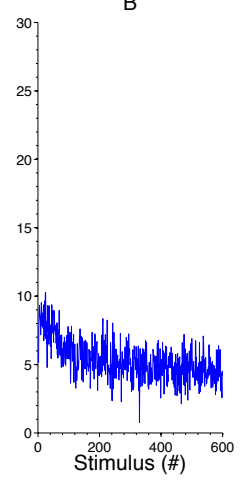

C

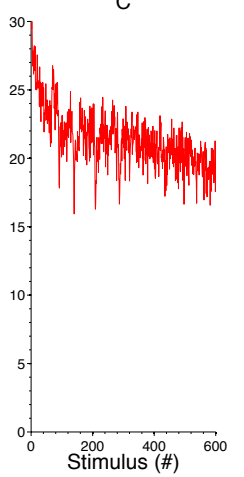

Fig. 4. Rapid C fiber accommodation to constant stimulation. (A) A constant stimulus charge of $100 \mathrm{nC} / \mathrm{Ph}$ was applied at $20 \mathrm{~Hz}$ for $30 \mathrm{~s}$. The insets show the pulse duration and amplitude of each stimulus $\left(I_{s t}=0.2 \mathrm{~mA} ; t_{s t}=0.5 \mathrm{~ms}\right)$. (B) Measured A $\gamma, \mathrm{B}$ and C peak response latencies relative to stimulus onset (ms). (C) Measured A $\gamma, B$ and C fiber peak response amplitudes relative to baseline $(\mu \mathrm{V})$. (D) Clustered stimulus response data from $B$-C. Amplitude and latency values associated with the second deflection of the diphasic fiber responses are also plotted in grey. (E) A color map representation of all stimulus response data shown in $A-C\left(N_{C N A P}=600 \mathrm{CNAP}\right.$ responses). Stimulus onset occurs at the intersection of the Stimulus \# and $t_{C N A P}$ axes. Note the significant increase in C peak latency and decrease in response voltage as Stimulus \# increases. (F) Fiber peak voltage as a function of stimulus number. A $\gamma$ fibers become slightly more excitable, as inferred from a general increase in the peak amplitude over the 600 stimuli. B and C fibers show significantly less activation as stimulus number increases.

shows another example of activation loss using 10x less charge per phase. In both cases, the $\mathrm{A} \gamma, \mathrm{B}$ and $\mathrm{C}$ fiber CNAP responses rapidly evolve over 30-s of constant current stimulation (see esp. Fig. 4E-F).

Linear regression was performed in STATA 12 to test for a statistically significant order effect, a characteristic feature of SIDNE [3]. A regression model slope coefficient that is significantly different from 0 suggests a relationship/change among the CNAP features of interest (i.e., $\mathrm{A} \gamma^{+}, \mathrm{B}^{+}$, or $\mathrm{C}^{+}$) and stimulus number (i.e., the sequential number assigned to each cathodal stimulus pulse within the 30 -s train of stimuli delivered at $20 \mathrm{~Hz}$ ). Significance tests of the slope coefficients suggest that, with an increasing number of stimuli, A $\gamma$ fiber excitability increases $\left(p\right.$-value $\left.=4.82 \mathrm{E}^{-9}\right)$ and $\mathrm{C}$ fiber excitability decreases $\left(p\right.$-value $\left.=5.34 \mathrm{E}^{-74}\right)$. A change in stimulus-driven nerve activation suggests an analogous change in output to tissues innervated by the nerve.

\section{B. Autonomous Stimulus-Response Measurement and Classification}

Fig. 5 summarizes a set of stimulus-response data that ANC collected from $\mathrm{C}$ fibers in the left cervical vagus nerve of the same rat whose data is represented in Fig. 4 and
Supplementary Fig. $1\left(P R F=20 \mathrm{~Hz} ; t_{\text {train }}=1 \mathrm{~s}\right.$; Conduction Distance $=8 \mathrm{~mm} ; N_{C N A P}=66$ trials x $20 \mathrm{CNAP}$ responses $/$ trial $=1320$ CNAP responses). Fig. 5A-C shows all $1320 \mathrm{~A} \gamma, \mathrm{B}$, and $\mathrm{C}$ fiber $\mathrm{CNAP}$ response latencies and voltages $\left(N_{\text {parameter }}=\right.$ 20 responses per stimulus parameter combination; $N_{\text {combo }}=66$ unique parameter combinations). Although $\mathrm{A} \gamma$ and $\mathrm{B}$ fiber stimulus-response data is collected, all stimulus intensity adjustments are based on the magnitude of the mean $\mathrm{C}$ fiber response in relation to the maximal response voltage. In Fig. 5D, data from Fig. 5A-C is clustered by response voltage and latency. Local minima from each fiber group are also plotted to demonstrate that other features of the CNAP response, such as peak-to-peak voltage and area, can be measured and used by ANC. A color map of all 1320 CNAP responses is shown in Fig. 5E (voltage is represented as a color according to the scale to the right of the figure). Finally, the mean CNAP responses are plotted by trial in Fig. 5F. Mean peak latencies are computed from the latencies of each individual stimulus response shown in Fig. 5A-E.

Suppl. Figs. 2 and 3 summarize data sets collected from A $\gamma$ and B fibers in the same nerve of the same subject (Suppl. Figs. 4-5 show data sets collected for $\mathrm{C}$ fibers in the same nerve of the same subject using a PRF of 2 and $1 \mathrm{~Hz}$, respectively). Note how the mean $\mathrm{C}$ fiber response magnitude 


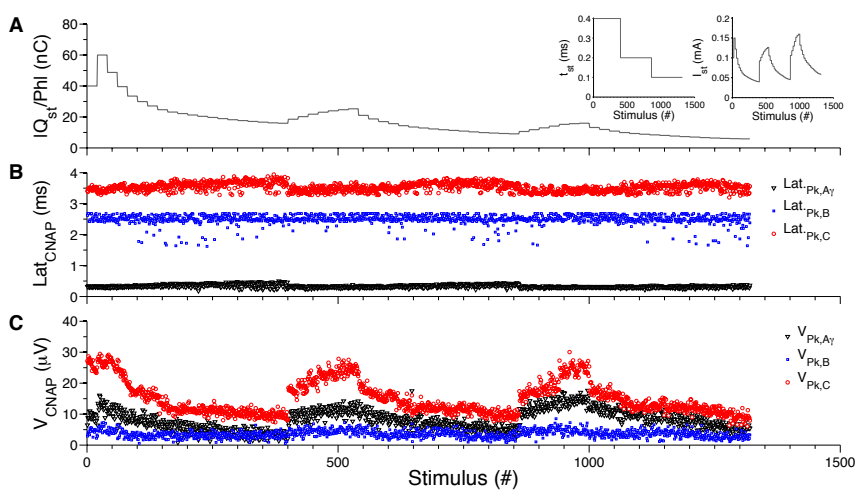

E

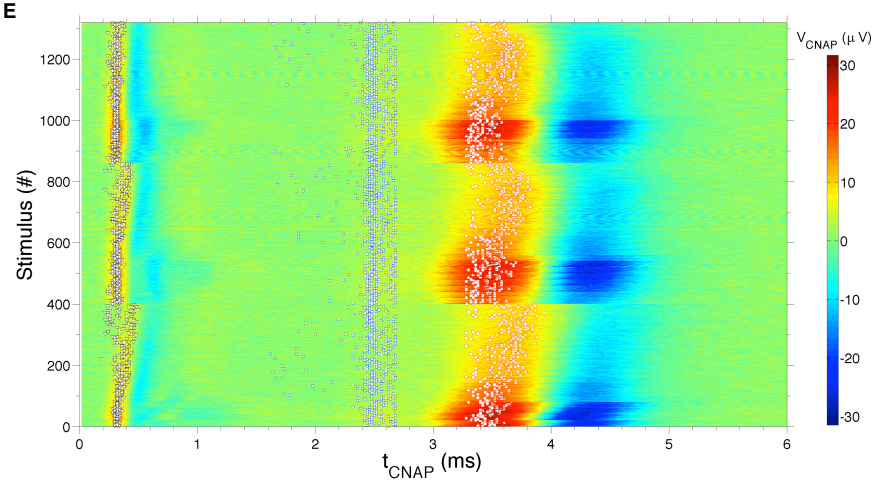

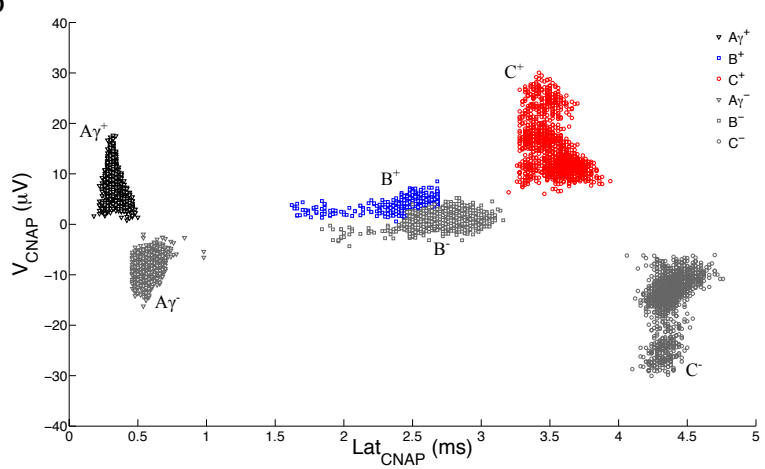

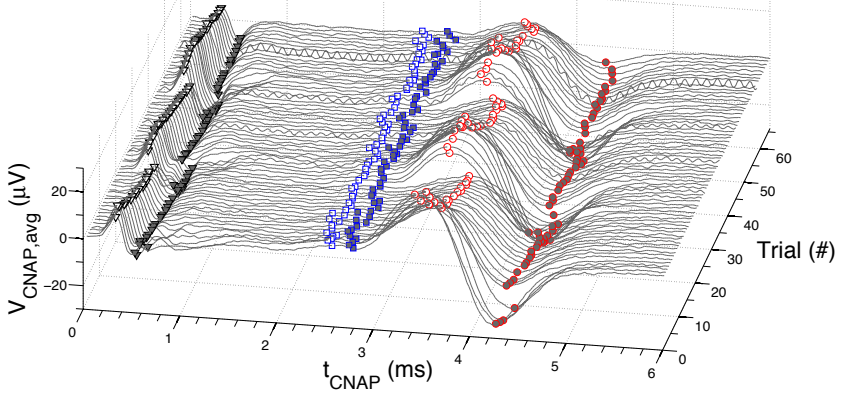

Fig. 5. Autonomously collected stimulus-response data from the left cervical vagus nerve of a female Long-Evans rat. (A) Stimulus charge per phase, in $\mathrm{nC} / \mathrm{Ph}$, for the entire vagal C-fiber activation profile-mapping period (ANC constructs a unique activation profile for each stimulated nerve and neuron type; the activation profile serves as a guide when maintaining or adjusting nerve activation for an experimental or therapeutic purpose). The insets show the pulse duration and amplitude of each stimulus. (B) Measured A $\gamma, \mathrm{B}$ and $\mathrm{C}$ peak response latencies relative to stimulus onset (ms). Latency increases with decreasing stimulus intensity for $\mathrm{A} \gamma$ and $\mathrm{C}$ fibers, but not for B fibers. (C) Measured A $\gamma, \mathrm{B}$ and $\mathrm{C}$ fiber peak response amplitudes relative to baseline $(\mu \mathrm{V})$. (D) Clustered stimulus response data from $B$-C. Amplitude and latency values associated with the second deflection of the diphasic fiber responses are also plotted in grey. (E) A color map representation of all stimulus response data shown in $A-C\left(N_{C N A P}=66\right.$ trials x 20 CNAP responses/trial $=1320$ CNAP responses). Stimulus onset occurs at the intersection of the Stimulus \# and $t_{C N A P}$ axes. (F) Mean CNAP response computed from data collected during each trial of stimulation $\left(N_{C N A P, \text { avg }}=20 \mathrm{CNAP}\right.$ responses/trial). Stimulus onset is at the intersection of the $V_{C N A P \text {, avg }}$ and $t_{C N A P}$ axes.

varies with $Q_{s t}$ in Fig. 5, whereas the mean $\mathrm{A} \gamma$ and B fiber response magnitudes vary with $Q_{s t}$ in Suppl. Figs. 2-3, respectively. The preferential modulation of $\mathrm{A} \gamma, \mathrm{B}$, or $\mathrm{C}$ fiber groups implies that the activation level of any fiber group in the nerve can be selectively controlled if the appropriate set of stimulus parameters are known.

\section{The Slope-Activation Relationship}

ANC rapidly identifies the parameter space for each fiber group in a nerve in the form of an activation profile. An activation profile is autonomously constructed for each fiber type using measured stimulus-response data and a newly discovered mathematical formula that relates threshold current (i.e., rheobase current) to fiber activation level. A set of activation profiles for each fiber group in a nerve constitutes a nerve activation profile.

The key to constructing a NAP is in a newly discovered, predictable relationship between the rheobase current, $I_{R h}$, and its corresponding fiber activation level, $\lambda\left(I_{R h}\right.$ is the slope of a charge-duration (CD) line described by the Weiss equation). When all possible CD lines are constructed from a set of stimulus-response data, each will represent a unique activation level, $\lambda$. If the slope of each line (i.e., $I_{R h}$ ) is plotted against its corresponding activation level, $\lambda$, an exponential slopeactivation relationship is observed. It is unique to each subject, nerve and neuron type, and allows ANC to adapt to changes at the device-tissue interface over the course of an experiment or therapy. To our knowledge, this is the first discovery and documentation of the relationship.

To derive the slope-activation relationship for vagal $\mathrm{A} \gamma, \mathrm{B}$ and $\mathrm{C}$ fibers, ANC first sorts the stimulus-response data in ascending order by the evoked response voltage. Each fiber response voltage is then normalized with respect to the maximal recorded response voltage and converted to a percentage of maximal activation. The largest observed response voltage represents maximal activation. All associated stimulus parameters are stored along with the measured nerve responses.

Next, ANC clusters the evoked fiber responses and associated stimulus parameters by activation level (a 5\% error tolerance is used by default). Within each cluster, the data is sorted by pulse duration (i.e., $t_{s t}$ ). If multiple entries have the same pulse duration and evoke the same level of activation, they are replaced with an average of the duplicate entries. ANC then searches for clusters with at least 2 pulse durations 
represented. Using these data, ANC computes the best-fit CD lines using least-squares linear regression.

The slope of each computed $\mathrm{CD}$ line (i.e., $I_{R h}$ ) is plotted against the associated percent maximal fiber activation, $\lambda$. To model the slope-activation relationships, ANC first computes the natural logarithm of each slope. Then, the best linear fit to the natural logarithm-transformed data is computed using least-squares linear regression. The resulting equation has the form $\hat{I}_{R h}=a r^{\lambda}$, where $\lambda=V_{\text {trial }} / V_{\max } \cdot 100$ is the percent maximal activation, $a$ is the predicted slope of the $\mathrm{CD}$ line for $0 \%$ maximal activation, and $r$ is a constant that determines the rate at which the slope of a $\mathrm{CD}$ line increases as activation level increases. In linear form, the slope-activation equation is $\ln \left(\hat{I}_{R h}\right)=\lambda \ln (r)+\ln (a)$. If $M=\ln (r)$ and $B=\ln (a)$, then $\hat{I}_{R h}=e^{M \lambda+B}$, where $M$ is the slope, $B$ is the y-intercept (i.e., the threshold current for $0 \%$ maximal activation), and $\lambda$ is the percent maximal activation. This can be used in place of $I_{R h}$ within the Weiss equation. In doing so, an equation that can be used to predict how a target fiber group will respond to any strength of ENS is created (2). The Weiss equation is shown above (2) for reference.

$$
\begin{aligned}
& \text { Weiss : } \frac{1}{t_{s t}} \int_{0}^{t_{s t}} I_{s t} d T=\frac{I_{R h}\left(t_{s t}+\tau_{S D}\right)}{t_{s t}}=\bar{I}_{s t} \\
& N A P: \frac{1}{t_{s t}} \int_{0}^{t_{s t}} I_{s t} d T=\frac{e^{M \lambda+B}\left(t_{s t}+\bar{\tau}_{S D}\right)}{t_{s t}}=\bar{I}_{s t}
\end{aligned}
$$

Fig. 6 shows the slope-activation data along with the associated equations and goodness-of-fit metrics for vagal $\mathrm{A} \gamma$, $\mathrm{B}$ and $\mathrm{C}$ fibers (derived using stimulus response data from Fig. 5 and Supplementary Figs. 2-3). The slope-activation data for A $\gamma$ fibers is least variable $\left(R^{2}=0.98\right)$, followed by $\mathrm{C}\left(R^{2}=\right.$ $0.86)$ and then B fibers $\left(R^{2}=0.36\right)$. A consistently poor signal- to-noise ratio is likely to blame for the poor fit to B fiber slope-activation data. A poor fit to the slope-activation data will translate to larger predictive errors once the activation maintenance mode of ANC is initiated. The model will evolve as ANC collects more data, however. Erroneous or inaccurate values in the slope-activation relationship are replaced once ANC locates the stimulus parameters that yield precisely the desired response. A simple voltage clamp enables ANC to rapidly and precisely locate the true parameter set that evokes the target response.

\section{The Nerve Activation Profile}

The activation profile for each fiber type is formed from the slope-activation equation and an estimate of the SD time constant, $\tau_{S D}$. The absolute value of the mean of the $\mathrm{x}$ intercept values from the CD lines is used as an estimate of $\tau_{S D}$. Given the subject, nerve, and fiber-specific constants $M$, $B$, and $\tau_{S D}$, (2) predicts the population response of any nerve fiber group to any strength of constant-current stimulation. This unique attribute is especially evident when solved for $\lambda$ in (3).

$$
\lambda=\frac{1}{M}\left[\ln \left(\frac{\bar{I}_{s t} \cdot t_{s t}}{t_{s t}+\bar{\tau}_{S D}}\right)-B\right]
$$

Fig. 7 graphically depicts the activation profiles that ANC constructed for vagal $\mathrm{A} \gamma, \mathrm{B}$ and $\mathrm{C}$ fibers using (2). The activation profile can be expressed in $\mathrm{CD}$ (top row) or SD form (bottom row). When the activation profile from each fiber group is overlaid, the NAP is complete. The NAP in Fig. 7 describes how the left cervical vagus nerve of one particular rat will respond to any strength of electrical stimulation. To aid interpretation, predicted CD lines (top row) and SD curves (bottom row) are shown within the parameter space for $[0,10$, $20, \ldots, 100] \%$ maximal activation. Note the nonlinear increase
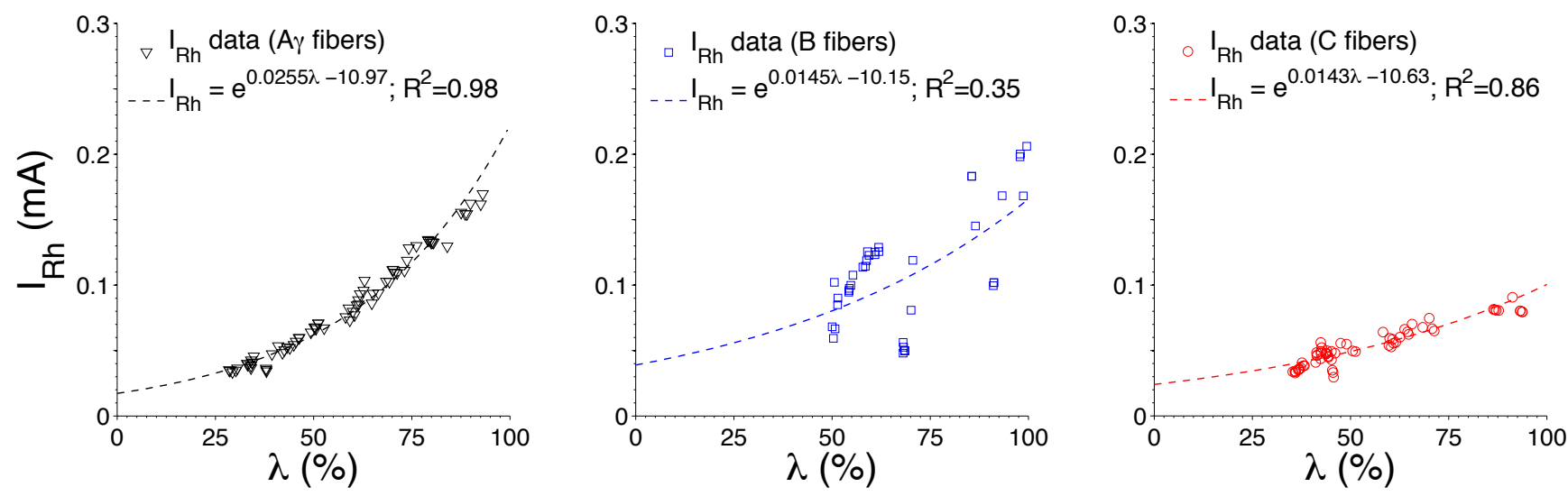

Fig. 6. Slope-activation data for A $\gamma$ (left), B (middle) and C fibers (right) for a single animal. For each fiber group, the rheobase current, $I_{R h}$, is plotted against its corresponding level of maximal activation, $\lambda$. Data shows an exponential increase in rheobase current for a linear increase in percent maximal activation. Best-fit curves are calculated for each fiber type through a least-squares linear regression of the natural logarithm-transformed slope-activation data. The coefficients $M$ and $B$ of the slope-activation equation are placed in the generalized form of the Weiss equation, producing a single equation that predicts how the target fiber type will response to any strength of stimulation. The goodness-of-fit is best for $A \gamma$ fibers $\left(R^{2}=\right.$ $0.98)$, followed by $\mathrm{C}$ and $\mathrm{B}$ fibers $\left(\mathrm{R}^{2}=0.86\right.$ and 0.35 , respectively). A poor fit is most closely associated with a poor signal-to-noise ratio. 

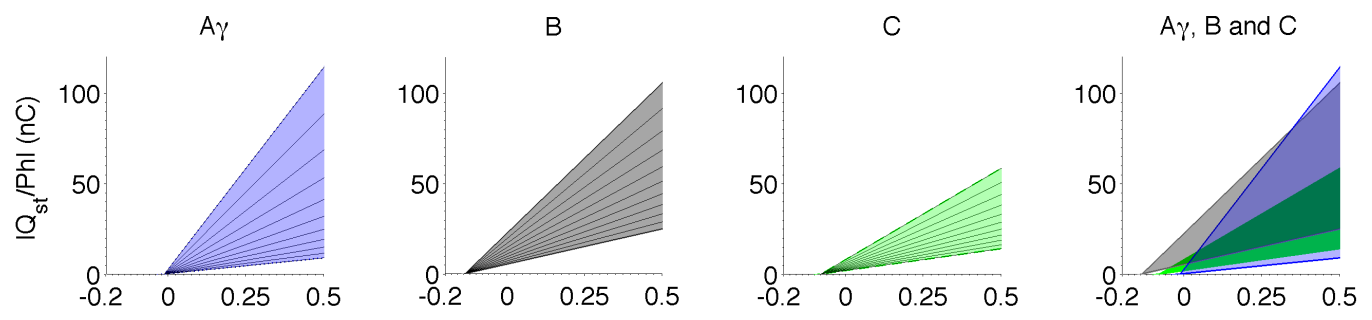

Fig. 7. Activation profile for $\mathrm{A} \gamma$ (left), B (middleleft) and $C$ (middle-right) fibers with predicted $\mathrm{CD}$ lines (top row) and SD curves (bottom row) for 0 to $100 \%$ maximal activation, in $10 \%$ increments. The right column shows the NAP, which predicts how all
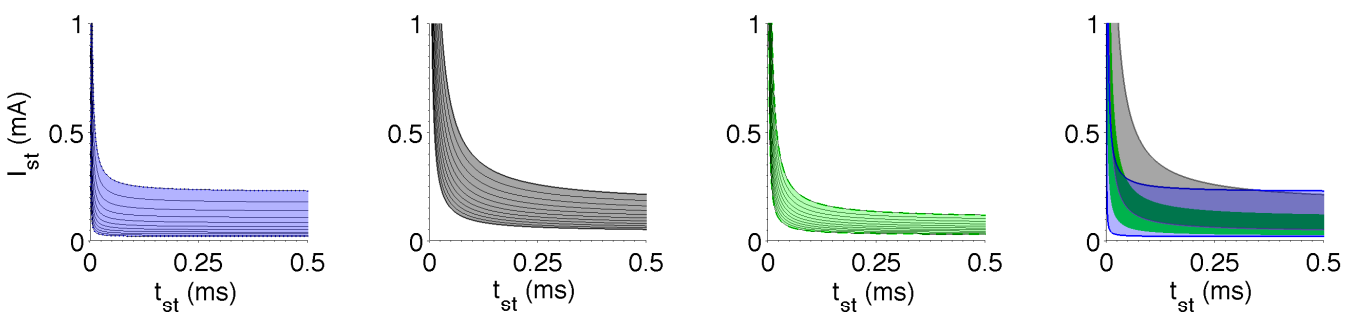

$\mathrm{A} \gamma, \mathrm{B}$ and $\mathrm{C}$ fibers in the nerve will respond to any strength of stimulation.

in slope with a linear increase in percent maximal activation, a property described by the coefficient $M$ in the slope-activation equation.

14]. Since the biological response to stimulation is not measured (neural activation or other biomarker), the stimulus parameters cannot be adjusted to maintain a constant degree of neural activation or therapeutic benefit over time.

\section{DISCUSSION}

ENS therapy holds the potential to modulate or control the function of almost every tissue in the body. Control is established by artificially modulating the firing activity of existing neural pathways with patterned electrical impulses from an implantable or external device. To maximize control over the quality of the therapy, the correct strength and pattern of ENS must be applied to selectively activate and control one or more specific neural pathways to deliver a therapeutic message to target tissues, organs, or systems, whose state is measured in the form of a biomarker response. Establishing control is problematic, as the degree of neural activation in response to a given dose of stimulus varies greatly from patient to patient (e.g., due to genetic differences, the tissue/immune response to the implant, or environmental factors) and changes over time in individual patients $[1,5,6]$. Although relevant biomarkers remain to be discovered for many conditions, the degree of nerve activation is a useful marker that can be beneficially exploited for the immediate advancement of bioelectronic therapies [12], therapies that require fine control over neural pathways for maximal therapeutic benefit.

All existing ENS technology fails to fully account for patient sensitivity to the electrical stimulus, especially at the nerve-electrode interface. To titrate the strength of ENS therapy today, a physician programs the stimulator to deliver a set of treatment parameters that describe the strength and pattern of electrical stimulation. Over a period of weeks to months, a physician slowly adjusts these parameters on a patient-to-patient basis in an attempt to maximize benefit and limit side effects [1]. The degree of nerve activation is not monitored or tracked in relation to therapeutic benefit, however, so the physician must rely on his/her past experience and patient reports for guidance. Months of subjectively guided treatment parameter adjustments can induce variable adverse side effects and significant patient discomfort $[5,13$,

ANC is a form of artificial intelligence that adjusts stimulus parameters in real time so that control is maintained over one or more neural pathways that mediate the target therapeutic effect and the off target effects (i.e., side effects). With the closed-loop, biofeedback-driven control provided by ANC, the degree of nerve fiber activation, ranging from 0 to $100 \%$, is controlled in the same manner across patients and within the same patient over time. ANC serves as a tool to advance our understanding of the relationships between the degree and pattern of neural activation and therapeutic efficacy. Moreover, it allows for the rapid if not immediate deployment of stimulus parameters that are optimized for each patient, nerve and neuron type. It is a new alternative to the long, burdensome device tuning system that is currently in use that can pave the way for a new standard of care.

\section{CONCLUSION}

ANC is device agnostic and modular in design, creating opportunities to develop additional subsystems that autonomously adjust the nerve activation level according to disease or stimulus-driven changes in the health of a patient (e.g., responsive or adaptive stimulation). Toward this end, ANC can accept a growing number of biological markers as input. Through collaboration, we expect to rapidly expand the capabilities of ANC and promote its use in the development of next-generation bioelectronic therapies. Under this paradigm, ANC will operate in tandem with secondary closed-loop systems, which instruct ANC when and how to activate select neuron populations in a nerve based on detected physiological events captured in the form of a biomarker (e.g., seizure onset). As a tool for unraveling the relationships between neural activation, biomarker changes and therapeutic benefit, ANC will further contribute to our understanding of treatmentresistant neurological diseases, their cause, their treatment and their prevention. 


\section{ACKNOWLEDGMENT}

We thank Cyberonics, Inc. for sponsoring work that led to the development of ANC. We also thank G. Albors, H. Desai, S.T. Lee, H. Zhang, J. Hunnicutt and M. Datta for the technical assistance that they provided. SWMJ is an Investigator of the Howard Hughes Medical Institute.

\section{REFERENCES}

[1] D. M. Labiner and G. L. Ahern, "Vagus nerve stimulation therapy in depression and epilepsy: therapeutic parameter settings," Acta Psychiatrica Scandinavica, vol. 115, pp. 23-33, 2007.

[2] B. M. Uthman, A. M. Reichl, J. C. Dean, S. Eisenschenk, R. Gilmore, S. Reid, et al., "Effectiveness of vagus nerve stimulation in epilepsy patients: a 12-year observation," Neurology, vol. 63, pp. 1124-1126, 2004.

[3] D. B. McCreery, T. G. H. Yuen, W. F. Agnew, and L. A. Bullara, "A characterization of the effects of neuronal excitability due to prolonged microstimulation with chronically implanted microelectrodes," IEEE Transactions on Biomedical Engineering, vol. 44, pp. 931-939, 1997.

[4] M. P. Ward, P. Rajdev, C. Ellison, and P. P. Irazoqui, "Toward a comparison of microelectrodes for acute and chronic recordings," Brain Research, vol. 1282, pp. 183-200, 2009.

[5] G. C. Albert, C. M. Cook, F. S. Prato, and A. W. Thomas, "Deep brain stimulation, vagal nerve stimulation and transcranial magnetic stimulation: An overview of stimulation parameters and neurotransmitter release," Neuroscience and Biobehavioral Reviews, vol. 33, pp. 10421060, 2009.

[6] W. M. Grill and T. J. Mortimer, "Stimulus waveforms for selective neural stimulation," IEEE Engineering in Medicine and Biology, pp. 375-385, 1995.

[7] D. B. McCreery, W. F. Agnew, T. G. Yuen, and L. Bullara, "Charge density and charge per phase as cofactors in neural injury induced by electrical stimulation," IEEE Transactions on Biomedical Engineering, vol. 37, pp. 996-1001, 1990.

[8] NationalResearchCouncil, Guide for the Care and Use of Laboratory Animals, 8th ed. Washington, D.C.: The National Academies Press, 2011.

[9] A. van Wieringen, O. Macherey, R. P. Carlyon, J. M. Deeks, and J. Wouters, "Alternative pulse shapes in electrical hearing," Hearing Research, vol. 242, pp. 154-163, 2008.

[10] M. D. Mann. (2011, May 2012). The Nervous System in Action. Available: http://michaeldmann.net/The Nervous System In Action.html

[11] D. M. Woodbury and J. W. Woodbury, "Effects of vagal stimulation on experimentally induced seizures in rats," Epilepsia, vol. 31, pp. S7-S19, 1990.

[12] K. Famm, B. Litt, K. J. Tracey, E. S. Boyden, and M. Slaoui, "A jumpstart for electroceuticals," Nature, vol. 496, pp. 159-161, 11 April 2013.

[13] M. S. George and G. Aston-Jones, "Noninvasive techniques for probing neurocircuitry and treating illness: Vagus nerve stimulation (VNS), transcranial magnetic stimulation (TMS) and transcranial direct current stimulation (tDCS)," Neuropsychopharmacology REVIEWS, vol. 35, pp. 301-316, 2010.

[14] L. B. Marangell, M. Martinez, R. A. Jurdi, and H. Zboyan, "Neurostimulation therapies in depression: A review of new modalities," Acta Psychiatrica Scandinavica, vol. 116, pp. 174-181, 2007.

[15] H. S. Gasser, "The Classification of Nerve Fibers," Ohio J Sci, vol. 41, pp. 145-159, 1941.

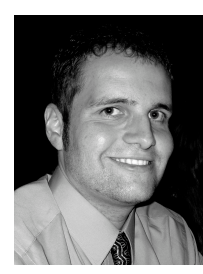

Matthew P. Ward was born in Port Shepstone, KwaZuluNatal, South Africa, in 1985. He received the B.S. degree in biomedical engineering and the Ph.D. degree in neuroengineering from Purdue University, West Lafayette, IN, in 2008 and 2012, respectively. From 2008 to 2012, he was a research assistant in the Center for Implantable Devices at Purdue University, developing closed-loop, device-based treatments for epilepsy and depression.

$\mathrm{He}$ is currently a research scientist in biomedical engineering at Purdue University, where he develops technologies to enable a persistent, bidirectional communication interface with the central and peripheral nervous system.

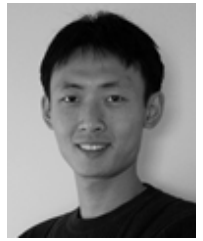

Kurt Y. Qing received the B.S. degree in biomedical engineering from Northwestern University in 2009. He is currently pursuing a combined M.D.-Ph.D. degree through the Medical Scientist Training Program at Indiana University School of Medicine (M.D. portion) and Purdue University ( $\mathrm{Ph}$. D. portion, in biomedical engineering).

From 2007 to 2009, he was an undergraduate research assistant at Northwestern University, Evanston, IL. Currently, he is working as a research assistant at Purdue University. His research interests include neurostimulation, biomedical instrumentation, and development of devices.

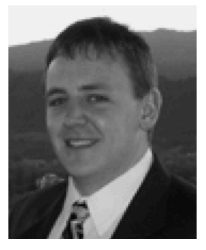

Kevin J. Otto (M'94) received the B.S. degree in chemical engineering from Colorado State University, Fort Collins, in 1997, and the M.S. and Ph.D. degrees in bioengineering from Arizona State University, Tempe, in 2002 and 2003, respectively.

From 2003 to 2006, he was a Post-Doctoral Fellow in the Department of Biomedical Engineering and the Department of Otolaryngology at the University of Michigan, Ann Arbor, where he focused on cochlear implants. He is currently an Associate Professor in the Department of Biological Sciences and Biomedical Engineering at the University of Florida, Gainesville. His research interests include neuroprostheses, systems neuroscience, and neurotechnologies.

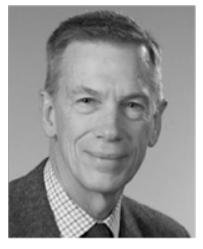

Robert M. Worth received his baccalaureate degree from Butler University, Indianapolis, IN, in 1963, and M.D. from Indiana University, Bloomington, IN, in 1966. He completed a general surgery residency internship at the Graduate Hospital of the University of Pennsylvania in 1967 before serving as a Flight Medical Officer in the US Air Force. He received the Ph.D. in biophysics from Indiana University in 1987 and the M.S. in mathematics from Purdue University in 2004.

Dr. Worth is currently a Professor of Neurological Surgery at the Indiana University School of Medicine and a Professor of Mathematical Sciences at the Indiana University-Purdue University of Indianapolis.

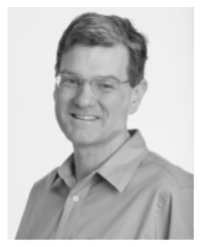

Simon W. M. John received the Joint Honors B.Sc. in zoology and genetics from the University College Cardiff, South Wales, UK, in 1985, and the Ph.D. in biology from McGill University, Montreal, Canada, in 1992.

He is currently an Investigator of the Howard Hughes Medical Institute and Professor at The Jackson Laboratory, Bar Harbor, ME. He is also a Research Assistant Professor in Ophthalmology at Tufts University School of Medicine and on the Graduate Faculty at the University of Maine. $\mathrm{He}$ is a world-renowned investigator of the molecular mechanisms of glaucoma.

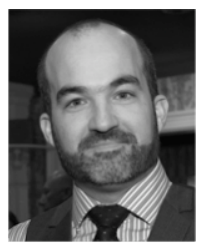

Pedro. P. Irazoqui (M'05) received the B.Sc. and M.Sc. degrees in electrical engineering from the University of New Hampshire, Durham, in 1997 and 1999, respectively, and the Ph.D. degree in neuroengineering from the University of California, Los Angeles, in 2003 for work on the design, manufacture, and packaging of implantable integrated circuits for wireless neural recording.

Currently, he is the Associate Head for Research and Associate Professor of biomedical engineering and an Associate Professor of Electrical and Computer Engineering at Purdue University, West Lafayette, IN. He has been Associate Editor of the IEEE Transactions on Biomedical Engineering since late 2006. 